\title{
EPILEPSIA EM PACIENTES COM MAIS DE 11 ANOS DE IDADE. CONSIDERAÇÕES A RESPEITO DE 1.000 CASOS COM ESTUDO CLÍINICO E ELETRENCEFALOGRÁFICO
}

\author{
Paulo Pinto Pupo \\ Olavo Pazzanese \\ B. A. Sampaio
}

A alta incidência de pacientes com crises convulsivas justifica todo o esfôrço para examiná-los em seus mais variados aspectos, sendo da máxima importância para o diagnóstico e para a orientação terapêutica, os elementos fornecidos pelos dados clínicos correlacionados com os exames subsidiários, maxime aquêles proporcionados pela eletrencefalografia.

A sistematização de uma rotina para o exame dos pacientes portadores de manifestações do tipo epiléptico, segundo os moldes preconizados pela escola neurológica de Montreal ${ }^{1,2}$, utilizada desde 1950 , vem permitindo obter resultados sumamente interessantes. No presente trabalho apresentaremos os resultados referentes a 1.000 pacientes observados, em sua grande maioria, em ambulatório ou em clínica privada. Os exames a que foram submetidos são, portanto, os de rotina clínica e eletrencefalográfica, e os resultados refletem aquilo que se pode obter na clínica cotidiana.

\section{MATERIAL E METODO}

A seleção dos pacientes obedeceu a critério exclusivamente clínico, de modo a que tivéssemos indicação segura de que eram portadores de manifestações de caráter epiléptico e elementos para reconhecer o tipo dessas manifestações. Os 1.000 pacientes foram selecionados de um grupo de 4.700 outros, com queixa clínica suspeita de epilepsia.

De todos foi feita anamnese detalhada quanto ao tipo de crise, sôbre os antecedentes pessoais (em particular antecedentes obstétricos, traumas ou moléstias outras afetando o encéfalo), os heredo-familiares, assim como sôbre a idade de início de suas perturbações. Cumpre assinalar que, como início da moléstia, foi considerado o momento em que ocorreu a primeira convulsão, mesmo que entre esta $\mathrm{e}$ as demais tenha havido longo intervalo.

Aos dados de anamnese foram acrescentados os do exame clínico, psiquiátrico e neurológico, assim como os do exame do líqüido cefalorraqueano.

Depois disso, foi feito o exame eletrencefalográfico, usando aparêlho Grass, modêlo III, com 8 canais. Usamos traçado inicial com referência comum (orelhas; e 16 eletrodos de rotina, seguido de tomadas bipolares sucessivas em linha ântero-

'Trabalho apresentadc na reunião da Sociedade Sul-Americana de Eletrencefalografia e Neurofisiologia Clínica, realizada em Lima (Peru), em 17 abril 1953 
posterior e em planos transversos, depois do que tomamos novo traçado con referência comum com eletrodo no vértex. Nesta tomada fazemos sistemàticaminte hiperventilação pulmonar por 3 minutos, seguida de repetição da mesma prova caso não se haja evidenciado elementos eletrencefalográficos de diagnóstico. A ativação pelo sono barbitúrico, assim como pelo metrazol, tem sido feita mais recentemente, em todos os casos em que o exame simples e com a hipernéia nada revelaram. Lm grande número de casos, após essa sequência de exames, fizemos também exames radiológicos do crânio (craniograma simples, pneumencefalograma ou arteriograma).

$O$ conjunto dos resultados clínicos e eletrencefalográficos foi projetado em mapas comparativos*, dos quais tiramos os elementos estatísticos para a organizaçãe dos quadros que seguem.

Inicialmente os pacientes foram separados por idades, em três grupos, respectivamente, de 11 a 20 anos, de 21 a 35 e de 36 a mais. Nos mapas os casos foram projetados individualmente, relacionando-se $\mathrm{em}$ colunas sucessivas os dados clínicos relativos a sexo, idade, idade de início da moléstia, tipo clínico da crise, antecedentes hereditários, antecedentes pessoais, resultados dos exames clínico, neurológico e psiquiátrico, resultados" dos exames do líqüido cefalorraqueano e radiológico.

Nas colunas seguintes foram dispostos os dados eletrencefalográficos segundo os tipos de alterações evidenciadas. Assim, consideramos: a) distúrbios indicativos da existência de processo lesional cerebral, difuso ou com foco convulsiógeno ativo, assinalando sua topografia; b) disritmias paroxísticas bilaterais síncronas (complexo ondas e espículas; disritmias por ondas $3 \mathrm{c} / \mathrm{s}$; hipersincronias, particularmente as com freqüência em tôrno de $6 \mathrm{c} / \mathrm{s}$ ); c) casos em que o eletrencefalograma não revelou sinais definidos de atividade convulsiógena.

Nas duas colunas finais colocamos os resultados da ativação pelo sono barbitúrico e da ativação pelo metrazol.

\section{RESULTADOS}

Us gráficos e quadros que apresentamos a seguir sintetizam os dados clínicos comparados aos eletrencefalográficos e vice-versa. Serão analisados de modo sumário, procurando-se pôr em destaque os elementos mais sugestivos de diagnóstico por êles apresentados.

O gráfico 1 resume os resultados eletrencefalográficos, verificando-se que, em 1.000 casos, $368(36,8 \%)$ mostraram sinais de lesão cerebral adquirida como fator causal; $325(32,5 \%)$ evidenciaram fatôres endógenos predominantes, portanto possível epilepsia idiopática; $307(30,7 \%)$ não revelaram sinais característicos de atividade convulsiógena. Os dados eletrencefalográficos mostram, pois, ligeira predominância dos fatôres causais de tipo lesional sôbre as predisposições endógenas.

Segundo a topografia, o lobo temporal foi o mais frequientemente afetado (154 em 368 casos), seguindo-se os focos profundos (86 casos). Entre êstes últimos figuram todos os focos subcorticais com projeção das disritmias em áreas extensas da superfície, podendo estar entre êles outros casos de foco temporal de situação mesial ou em pontos de passagem para a ínsula de Reil, o que aumenta ainda mais o número de possíveis focos de situação temporal. Seguem-se, em frequiên-

* Para maior compreensão. veja-se mapa similar apresentado por Pupo, Pazzanese

e Sampaio ${ }^{3}$. 


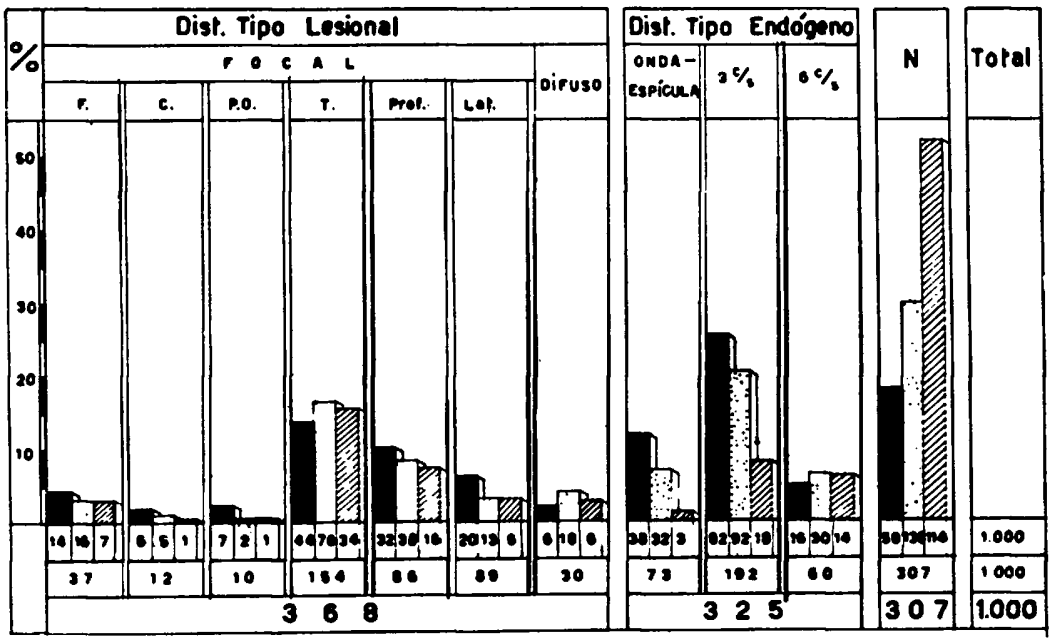

Gráfico 1 - Resultados eletrencefalográficos em 1.000 pacientes.

Gráfics 2 - Resultados do eletrencefalograma segundo os grupos etários.

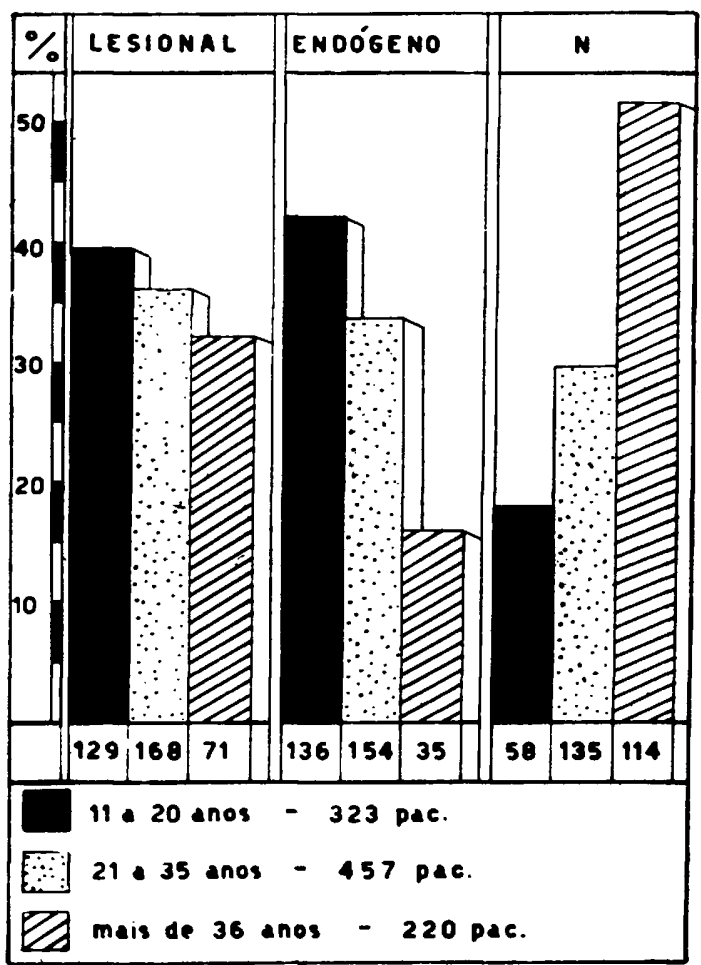


cia, os focos frontais ( 37 casos) e os das circunvoluções centrais (12 casos). Apenas 10 casos apresentaram focos situados na extensa zona parieto-occipital, comprovando a pequena tenciência destas áreas a reagir convulsivamente.

Nas alterações eletrencefalográficas do tipo endógeno destacam-se as disritmias por ondas $3 \mathrm{c} / \mathrm{s}$ bilaterais e síncronas (192 em 325 casos).

Os resultados não característicos $(\mathrm{N})$ serão analisados adiante.

$O$ gráfico 2, que resume o anterior, tem a finalidade de relacionar os resultados eletrencefalográficos com os grupos etários. Vemos que os fatôres lesionais se apresentam de forma equilibrada nos 3 grupos, predominando ligeiramente no grupo de 11 a 20 anos. Eles aparecem em $39,8 \%$ dos casos no primeiro grupo, $36,75 \%$ no segundo e $32,3 \%$ no terceiro. Quanto aos fatôres endógenos, verificamos que decrescem progressivamente com a idade, incidindo numa pequena percentagem nos indivíduos de mais de 35 anos (16\%).

Com respeito aos resultados não característicos notamos, ao contrário, que sua percentagem aumenta ̀̀ medida que a idade avança, sendo que, no grupo com mais de 35 anos isto mais se evidencia $(57,4 \%$ dos casos). Êstes dois últimos itens serão melhor estudados no gráfico 3 .

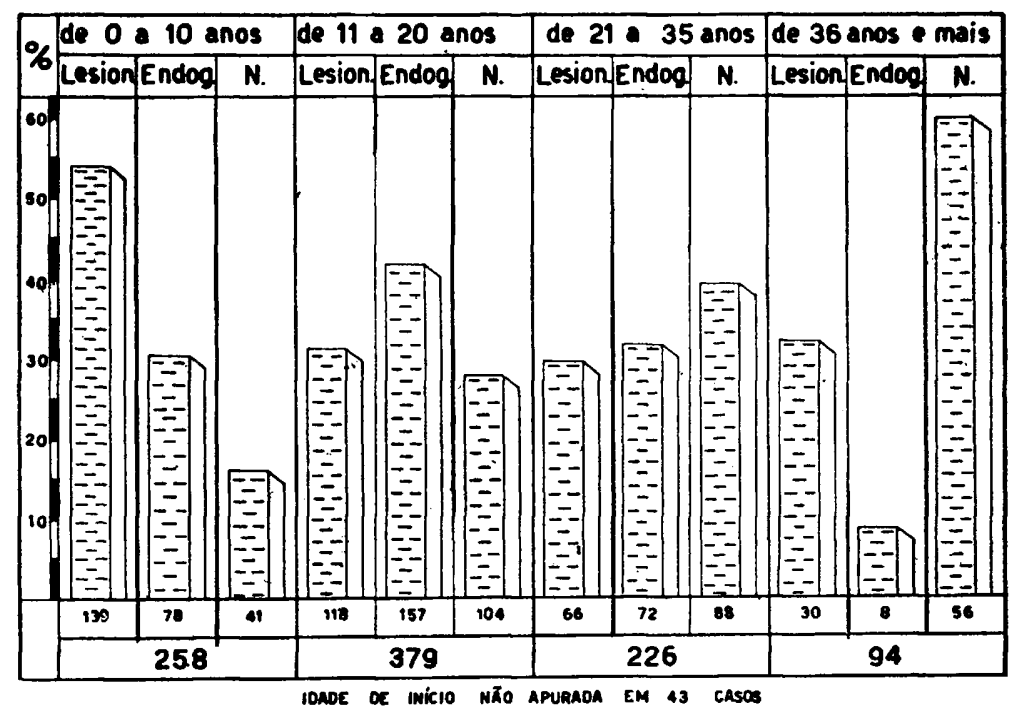

Gráfico 3 - Correlação entre tipos de resultados eletrencefalográficos segundo a idade de início da moléstia.

O gráfico 3 correlaciona os resultados eletrencefalográficos com o tempo de início da moléstia; êste dado não foi possível obter em 43 casos. Consideramos como idade de início aquela que o paciente tinha quando sofreu sua primeira crise convulsiva, mesmo que houvesse um intervalo de anos entre esta $\mathrm{e}$ as que motivaram a consulta.

Nos pacientes cuja doença se iniciou numa idade inferior a 10 anos, predominaram os fatôres causais adquiridos. Neste grupo tivemos, para 139 lesionais, 79 
endógenos. Em números percentuais: 53,9\% de lesionais; 30,2\% com fatôres endógenos predominantes; $15,9 \%$ sem sinais eletrencefalográficos característicos.

Êstes dados intèressam bastante porque concordam com os que obtivemos em pacientes com menos de 11 anos de idad 23 (52,9\% de fatôres causais lesionais; $29,4 \%$ de tipo endógeno predominante; $17,7 \%$ sem distúrbios característicos). Esta coincidência certifica quanto à precisão de nossas pesquisas anamnésticas e, por outro lado, destaca o valor de se considerar a primeira crise como dado diagnóstico.

No grupo de pacientes com idade de início entre 11 e 20 anos, houve predominância dos fatôres endógenos $(41,5 \%)$, de acôrdo com as noções clássicas. No 4: grupo quanto à idade chama a atenção a pequena percentagem de casos com fatôres endógenos predominantes $(8,5 \%)$.

Examinando-se os grupos etiológicos em separado, veremos que o dos fatôres lesionais mantëm suas percentagens sem grandes modificações, a partir de 11 anos, enquanto que o dos fatôres endógenos atinge o máximo no segundo decênio e em seguida decresce. Reafirma-se o que já havíamos observado ao analisar o gráfico 2 .

Vejamos agora, em conjunto, os resultados não característicos (N).

Há aumento progressivo do número dêstes casos à medida que a idade avança $(15,9 \%$ no primeiro grupo, $27,5 \%$ no segundo, $39 \%$ no terceiro, $59,7 \%$ no quarto). O aumento do número de resultados não característicos é mais pronunciado em relação à idade de início do que à idade atual (compare-se com o gráfico 2). Esta grande percentagem de casos com eletrencefalograma sem alterações, entre pacientes maiores de 35 anos, está em desacôrdo com as noções clássicas, que afirmam serem as convulsões tardias muito mais sugestivas de foco lesional do que as demais. Eila sugere a probabilidade de serem essas convulsões tardias devidas mais a fatôres gerais que atuem no momento da crise, por exemplo, toxicoses (alcoolismo), distúrbios circulatórios locais devidos a arteriosclerose e hipoglicemia. A continuação das pesquisas nesse sentido poderá, no futuro, confirmar essa hipótese.

Possivelmente, o grande número dêstes pacientes com EEG negativo se deve a fatôres endógenos não revelados nos traçados; êsses fatôres endógenos, como é sabido, têm muito menor possibilidade de se evidenciarem $\mathrm{em}$ cérebros adultos. $A$ análise de nossos resultados sustenta êste raciocínio, evidenciando que os fatôres lesionais se mantêm equilibrados nos três grupos de idades. Ora, isto significa que o grupo com fatôres endógenos predominantes e o grupo com EEG sem alterações guardam entre si uma proporção constante. De fato, o número de pacientes com fatôres endógenos decresce com o progredir da idade, ao passo que o número daqueles com EEG normal cresce proporcionalmente no mesmo sentido, sugerindo que os casos dêstes dois grupos estão intimamente ligados e, possìvelmente são, em grande maioria, da mesma natureza. O tipo de crise clínica GiM predominantemente entre os pacientes com EEG sem alterações, como veremos no quadro 6, é uma contraprova dêste nosso raciocínio.

No quadro 1 comparamos os resultados eletrencefalográficos, respectivamente de tipo lesional e de tipo endógeno, com os elementos fornecidos pela clínica. Vemos que existe grande predominância das manifestações de crise clínica do tipo lesional coincidindo com as eletrencefalográficas, e que, em particular, o tipo destas manifestaçôes coincide com a topografia do foco convulsiógeno. Pelo contrário, as manifestações clínicas de tipo endógeno coincidem, na grande maioria, com as eletrencefalográficas.

A concordância de resultados clínicos e eletrencefalográficos vem dar ênfase à realidade de nossos resultados, e nos permite, daqui por diante, raciocínio em base, quer de dados clínicos, quer de dados eletrencefalográficos. 


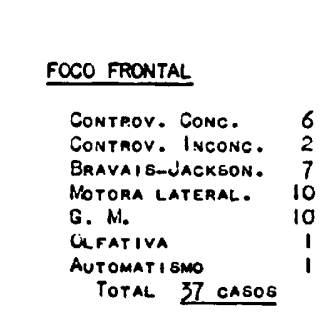

FCCC CENTRAL

Controv. InCONC. I Bravais-Jackson. 6 MOTOAA LATERAL. 1 G. N.

Automat Ismo

Total II CASOS

\section{FOCO PARIETO-OCIPITAL}

Controv. Conc.
Bravais-Jackson.
Motora lateral.
Auoit. vertig.
Total IO casos

\section{TIPO LESIONAL}

FOCO TEMPORAL

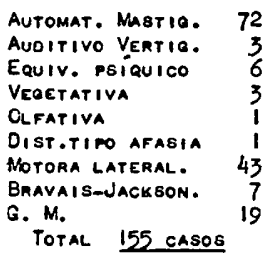

\section{FCCO LATERALIZADO}

Bravais-Jackson. 13 Motora lateral.

G. $M$.

P. M.

Automatismo

Equiv. Psíquico

Auditiva VERTIG.

Glativa

TOTAL 38 CAEOS

\section{FOCO PRORUNDO}

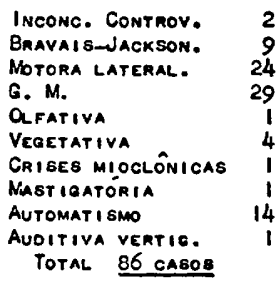

\section{ALTERACOEES OIFUSAS}

G. $M_{*}$

BRAVAI GadAGKSON. 3

TOTAL 30 CASOS

TOTAL OERAL

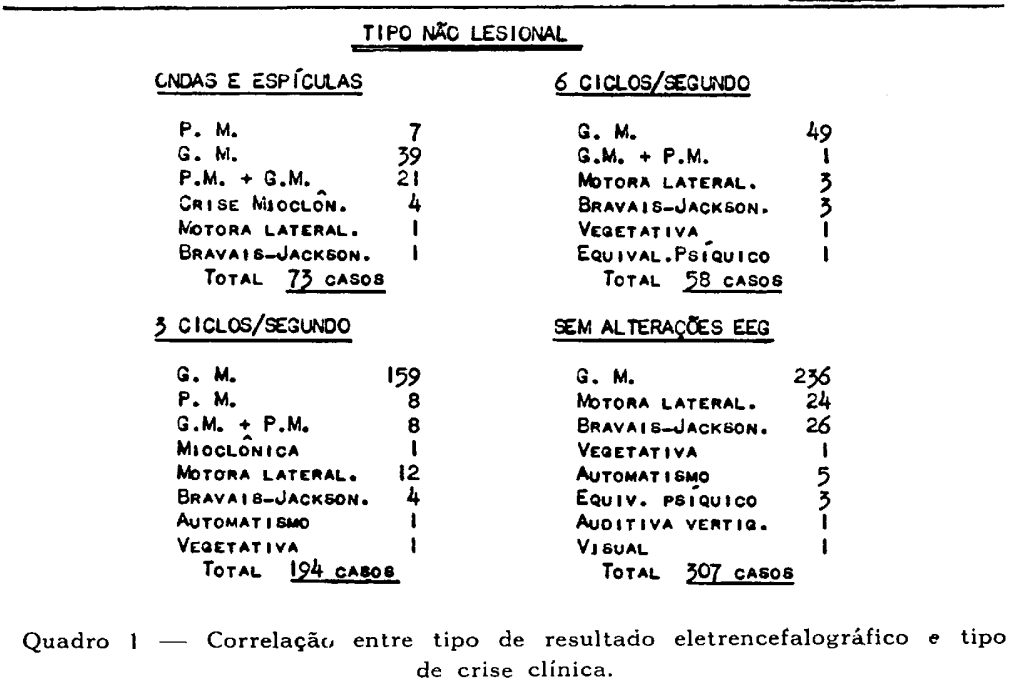

Num total de 120 pacientes, a grande maioria (93) apresentou distúrbios eletrencefalográficos de tipo lesional. Esta afirmação é verídica também para cada um dos grupos de idade separadamente. Há, pois, perfeita concordância clínicoeletrencefalográfica.

Como era de esperar, predominam as alterações do tipo endógeno sôbre as do tipo lesional (90:55). A predominância dêsses elementos no grupo de idade de 11 a 20 anos concorda com a predominância dos fatôres endógenos neste grupo, comparativamente com os demais.

A ativação pelo sono barbitúrico (quadro 4) foi feita nos casos em que o exame simples ou ativado pela hiperpnéia nada revelou. Usamos na rotina diária 


\begin{tabular}{|l|c|c|c|c|}
\hline TIPO DE EEG & 11 a 20 & 21 a 35 & Mals de 36 & TOTAL \\
\hline Lestonal & 39 & 36 & 13 & 93 \\
Alt. t1po endogeno & 7 & 4 & 0 & 11 \\
Serm alteraçōe s & 3 & 8 & 5 & 16 \\
\hline TOTAL & 49 & 48 & 23 & 120 \\
\hline
\end{tabular}

Quadro 2 - Casos com sindrome neuropsíquica de afecção orgânica cerebral (além das convulsões).

\begin{tabular}{|l|c|c|c|c|}
\hline TIPO DE BBO & 11 a 20 & 21 a 35 & mais de 36 & TOTAL \\
\hline Leslonal & 31 & 22 & 3 & 55 \\
Alt.tipo endogeno & 46 & 34 & 10 & 90 \\
Som altersçös & 12 & 36 & 26 & 74 \\
\hline TOTAL & 89 & 91 & 39 & 219 \\
\hline
\end{tabular}

Quadro 3 - Casos com hereditariedade similar.

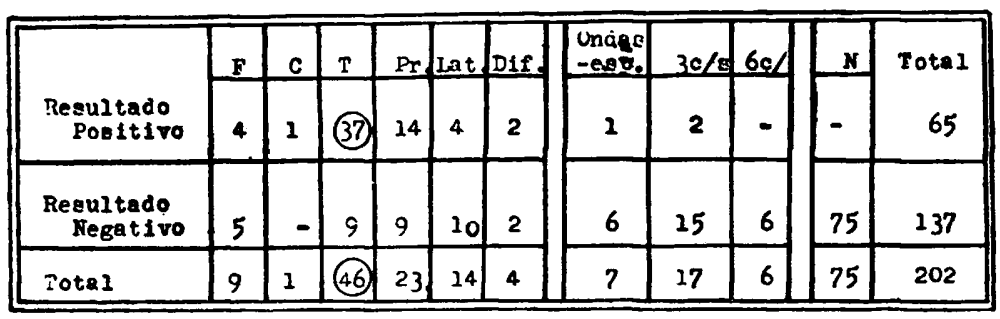

Quadro 4 - Resultados da ativação com o sono barbitúrico.

o Seconal sódico, por via oral, na dose de 100 a $300 \mathrm{mg}$, como meio mais prático de provocar o sono. Do total de 202 casos, conseguimos positivar elementos em 65. Entre os casos em que foi positiva a ativação sobressaem os de foco temporal, nos quais foi elucidativa em 37 e negativa sòmente em 9.

A ativação pelo metrazol (quadro 5) entrou para nossa rotina mais recentemente. $O$ método utilizado foi a injeção intravenosa da solução a $5 \%$ de metrazol, fracionada na dose de $50 \mathrm{mg}$ cada $30 \mathrm{seg}$, até provocar disritmias no traçado. Usamos a dose total limite de $500 \mathrm{mg}$ e excepcionalmente chegamos à crise clínica. Foi realizada em 55 pacientes, com o mesmo critério com que agimos na ativação 


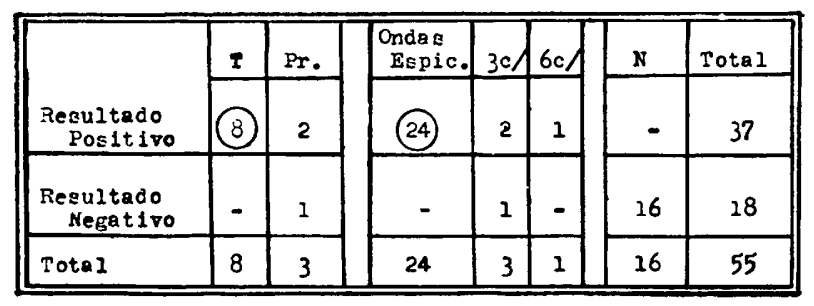

Quadro 5 - Resultados da ativação com o metrazol.

pelo sono. Obtivemos resultados positivos em 37 casos, sobressaindo-se os de complexo "onda e espícula", num total de 24 casos.

$O$ quadro 6 é o reverso do quadro 1: procura relacionar o tipo de crise clínica com os resultados eletrencefalográficos.

\begin{tabular}{|c|c|c|c|c|c|}
\hline $\begin{array}{l}\text { Idade do } \\
\text { Paciente }\end{array}$ & Q. Mo & P. $\mathrm{Mo}$ & $\begin{array}{l}\text { Bp11. } \\
\text { Temp. }\end{array}$ & $\begin{array}{l}\text { Eravais- } \\
\text { Jackson. }\end{array}$ & $\begin{array}{l}\text { Qutros tipos } \\
\text { Wotora wh. }\end{array}$ \\
\hline \multicolumn{6}{|c|}{ IRSIOMAL } \\
\hline $\begin{array}{c}11 \text { a } 20 \\
\text { enos }\end{array}$ & 28 & 0 & $x_{4}$ & 17 & 38 \\
\hline $21 \underset{\text { anos }}{35}$ & 39 & 1 & 61 & 23 & 38 \\
\hline $\begin{array}{l}\text { Mals de } \\
36 \text { anos }\end{array}$ & 19 & 0 & 30 & 10 & 12 \\
\hline Total & 86 & 1 & 115 & 50 & 88 \\
\hline \multicolumn{6}{|c|}{ ENDOGENO } \\
\hline $\operatorname{lnos}^{11: 20}$ & 95 & 27 & 4 & 1 & 9 \\
\hline 21 anos 35 & 122 & 16 & 0 & 4 & 6 \\
\hline $\begin{array}{l}\text { Mais do } \\
36 \text { anos }\end{array}$ & 35 & 0 & 0 & 0 & 0 \\
\hline Totgl & 252 & 43 & 4 & 5 & 15 \\
\hline \multicolumn{6}{|c|}{ NADA } \\
\hline $\begin{array}{l}11 \text { a } 20 \\
\text { anos }\end{array}$ & 46 & D & 2 & 4 & 5 \\
\hline 21 anos 35 & 98 & 0 & 4 & 20 & 13 \\
\hline $\begin{array}{l}\text { Ma 1s do } \\
36 \text { anos }\end{array}$ & 92 & 0 & 4 & 12 & 6 \\
\hline Total & 236 & 0 & 10 & 36 & 24 \\
\hline So $A$ & 574 & 44 & 129 & 91 & 127 \\
\hline
\end{tabular}

Quadro 6 - Correlação entre tipo de crise clínica a EEG. 
Vemes que, de 574 pacientes que apresentaram crises do tipo grande mal, 252 revelaram ao eletrencefalograma distúrbios endógenos, portanto, de acôrdo com o que era de esperar, e, em 86, o eletrencefalograma mostrou fatôres causais do tipo lesional. Chama a atenção, entretanto, o grande número de pacientes con convulsões do tipo grande mal nos quais o eletrencefalograma nada mostrou de característicos: um total da 236 casos. Não acreditamos que esta última verificação seja causada por êrro de interpretação diagnóstica, pois, se assim fôsse, êste êrro estaria distribuído equitativamente em todos os grupos, não havendo predominância nos dois últimos grupos de idade. Pensamos, antes, que grande número dêsses casos corresponde a pacientes com fatôres endógenos não evidenciáveis no eletrencefalograma, tal como afirmamos ao comentar o gráfico 3.

Já nos pacientes com diagnóstico de crises tipo pequeno mal, em 43 sôbre 44 o eletrencefalograma confirmou a natureza endógena da moléstia.

Nos portadores de crises epilépticas de tipo automatismo, ou com aura vegetativa, mastigatória, eqüivalentes psíquicos, olfativa, auditiva, isto é, do grupo epilepsia temporal, num total de 129, os fatôres lesionais foram demonstrados em، 115 casos; em 4 casos encontrou-se fatôres endógenos, provàvelmente devido a êrro de diagnóstico clínico, e, em 10, nada ficou demonstrado pelo eletrencefalograma. Como se vê, êste grupo permite grandes possibilidades diagnósticas.

Em relação ao grupo de crises tipo bravais-jacksoniano surpreendeu-nos o elevado número de casos $(36: 91)$ em que o eletrencefalograma não demonstrou sinais evidentes de focos convulsiógenos. E' uma observação em aparente discordância com os conceitos clássicos e que merece ser melhor estudada no futuro.

Na última coluna do quadro 6 reunimos os demais tipos clínicos de convulsões focais Usamos a denominação genérica de convulsões motoras generalizadas para aquêles casos em que a anamnese forneceu elementos de convulsão motora e de lateralização, se bem que não tivesse sido suficiente para afirmar se bravaisjacksoniana, controversiva, ou de outro tipo. Dentre 127 casos, 88 apresentavam distúrbios eletrencefalográficos de tipo lesional, como era de esperar.

Nossos resultados provêm, reafirmamos, de pacientes de ambulatório e de clínica privada. Nêles não pudemos utilizar intensamente os métodos de ativação eletrencefalográfica, nem mesmo repetir os exames, quando negativos. A grande maioria foi submetida sòmente a um exame eletrencefalográfico. Isto explica a divergência existente entre os nossos $30,7 \%$ de resultados negativos, comparativamente a outras estatísticas da literatura. Comparada, por exemplo, com as de Kershman, Vásquez e Goldstein $^{4}$ vemos sensivel disparidade neste grupo. Mas, nessas mesmas estatísticas vamos encontrar a grande influência exercida sôbre os resultados pelo tipo de exame empregado, se em pacientes levados a um laboratório de pesquisas ou se de pacientes de ambulatório. Assim, vemos que, entre os militares, cuidadosamente estudados, os autores encontraram $66 \%$ de atividade focal, enquanto entre os civis essa percentagem não foi além de $38 \%$. F.m relação aos tipos de alterações, lesionais ou bilaterais síncronas, por êles encontradas comparativamente com os grupos de idades, assim como a data de início da moléstia, nossas estatísticas são relativamente concordes. 


\section{RESUMO E CONCLUSOES}

Os autores fizeram revisão do estudo clínico e eletrencefalográfíco de 1.000 pacientes com manifestações clínicas do tipo epiléptico, selecionados entre 4.700 casos estudados.

Em todos os casos foi obtida anamnese detalhada sôbre o tipo de crise, os antecedentes pessoais (em particular antecedentes obstétricos, traumas ou moléstias outras afetando o encéfalo) e heredo-familiares, assim como a idade de início de suas perturbações. Cumpre assinalar que a primeira convulsão foi considerada como início da moléstia, mesmo quando entre ela e as demais tivesse havido um intervalo de anos. Paralelamente, em todos os casos foram feitos exames clínico, neurológico e psiquiátrico, e, em grande número dêles, exames de líqüido cefalorraqueano e radiográfico do crânio (craniograma simples, pneumencefalograma e arteriograma).

As manifestações clínicas, classificadas segundo o critério de Penfield e colaboradores, foram comparadas com os resultados obtidos pela eletrencefalografia (simples ou ativada) e finalmente projetadas em gráficos e quadros. Esstes foram catalogados separadamente segundo os grupos de idade de 11 a 20 anos, de 21 a 35 anos, e de 36 e mais.

Os resultados clínicos e eletrencefalográficos, assim como a compara. ção entre uns e outros, permitem destacar os seguintes fatos:

1. No total dos pacientes o eletrencefalograma mostrou alterações de tipo lesional em $36,8 \%$, alterações de tipo endógeno em $32,5 \%$ e não evidenciou alterações de tipo epiléptico em $30,7 \%$.

2. Os pacientes portadores de alterações do tipo lesional assim se distribuíram segundo os grupos de idade: $39,8 \%$ no primeiro grupo (11 a 20 anos): $36,7 \%$ no segundo (21 a 35 anos) e $32,30 \%$ no terceiro (36 e mais).

3. Estes mesmos pacientes assim se distribuíram segundo a idade do início da moléstia: 53,9\% no grupo de 0 a 10 anos, $31 \%$ no grupo de 11 a 20 anos. $29,1 \%$ no grupo de 21 a 35 anos e $31,8 \%$ no grupo de mais de 36 anos.

4. Os pacientes portadores de alterações do tipo cndógeno assim se distribuíram segundo os grupos de idade: $42,25 \%$ no primeiro grupo, $33,75 \%$ no segundo e $16 \%$ no terceiro.

5. Tais pacientes assim se distribuíram segundo a idade do início da moléstia: $30,2 \%$ no grupo de 0 a 10 anos, $41,5 \%$ no grupo de 11 a 20 anos, $31,9 \%$ no grupo de 21 a 35 anos e $8,5 \%$ no grupo de 36 anos e mais.

6. Os pacientes em que o EEG nada revelou assim se distribuíram segundo os grupos de idade: $17,94 \%$ no primeiro grupo, $29,50 \%$ no segundo e $51,70 \%$ no terceiro. 
7. Esses pacientes, segundo a idade do início da moléstia, assim se distribuíram: $15,9 \%$ no grupo de 0 a 10 anos, $27,5 \%$ no grupo de 11 a 20 anos, $39 \%$ no grupo de 21 a 35 anos, e, finalmente, 59,7\% no grupo de 36 anos e mais.

8. O tipo de distúrbio eletrencefalográfico, assim como a topografia das alterações focais, quando presentes, coincidem, em elevada percentagem de casos, com o tipo das manifestações clínicas dos pacientes.

9. As crises de tipo bravais-jacksoniano, em discordância com os demais tipos de crises focais, apresentou elevada proporção de casos com EEG negativo (36 em 91 casos). As crises de epilepsia temporal mostraram 10 casos negativos num total de 132 , e as demais crises motoras lateralizadas evidenciaram sòmente 18 num total de 125 casos.

10. A crise do tipo GM foi a que mais apresentou EEG negativo (236 num total de 572 casos). A crise de tipo PM confirmou integralmente o diagnóstico de fator endógeno predominante (43 sôbre 44 casos).

11. As crises de tipo temporal apresentaram, em grande proporção de casos, distúrbios de tipo lesional no EEG (118 num total de 132 casos) e, em particular, tais distúrbios lesionais foram de tipo focal nos lobos temporais (86 num total de 118 casos).

12. As demais crises motoras lateralizadas evidenciaram igualmente, em elevada proporção, distúrbios de tipo lesional no EEG (88 num total de 127 casos).

13. A presença de epilepsia em parentes até o terceiro grau mostrou coincidir com a maior proporção de casos de epilepsia endógena (90 num total de 219 pacientes).

14. Erri reverso, os sinais neuro-psiquiátricos de afecção orgânica cerebral coincidiram, em elevada proporção, com a presença de distúrbios de tipo lesional nos traçados eletrencefalográficos (93 num total de 120 pacientes).

15. A ativação pelo sono barbitúrico tem sido feita nos casos em que o exame simples ou ativado pela hiperpnéia nada revelara ou com finalidade de melhor identificar distúrbios frustros. Num total de 202 casos em que foi empregada, conseguiu positivar elementos em 65 . Entre os casos em que a ativação pelo sono foi positiva sobressaem os de crise temporal, nos quais foi elucidativa em 37, e negativa sòmente em 9.

16. A ativação pelo metrazol, usada de rotina mais recentemente, foi feita em 55 pacientes, com o mesmo critério. Ela conseguiu positivar o diagnóstico em 37.

17. E' de salientar que, dos 307 pacientes em que o EEG não evidenciou característicos de tipo epiléptico, percentualmente o maior número estava entre os de idade superior a 35 anos. Isto faz pensar na possível 
existência de outros fatôres correlativos (intoxicações crônicas, anóxia por arteriosclerose cerebral, hipoglicemia, etc.), que não os lesionais cerebrais, na gênese de seus distúrbios paroxísticos.

\section{SUMMARY AND CONCLUSIONS}

The authors made the review of a thousand of patients with clinical syndrome of epilepsy, in whom clinical and EEG studies were obtained. These one thousand cases were selected among 4,700 cases of epileptic patients with EEG diagnosis.

From all cases, detailed history of the type of seizure, of past history (particularly obstetrical history, traumatic lesions and other cerebral lesions), of familial history, and of the beginning of convulsive seizures, have been obtained. In every case the onset of the disease was considered as the first convulsive seizure, even when between the first and the next there was a large period of time. In every case clinical, neurological and psychiatric examinations were obtained. In many of the cases spinal fluid and radiological (craniogram, pneumoencephalogram and arteriogram) examinations were performed.

The clinical manifestations were classified according to Penfield and col. These clinical features were compared with the EEG records (in 202 cases with barbiturate sleep and in 55 cases with metrazol), and projected in graphs and tables. In these the patients were classified according to age in three different groups (from 11 to 20 years of age, from 21 to 35 , and with more than 36 years of age).

From this study, the authors call the attention to the following data:

1. The EEG showed changes of lesional type in 36.8 per cent of the cases, of endogenous type in 32.5 per cent and did not show epileptic changes in 30.7 per cent.

2. Patients with lesional type of EEG could be classified according to age in the following manner: 39.8 per cent in the first group (from 11 to 20 years of age), 36.75 per cent in the second group (from 21 to 35 years of age) and 32.30 per cent in the third group (with more than 36 years of age).

3. The same patients could be classified according to age of the first seizure: 53.9 per cent in the group from zero to 19 years, 31 per cent in the group from 11 to 20 years, 29.1 per cent in the group from 21 to 35 years, and 31.8 per cent in the group with more than 36 years.

4. Patients with endogenous changes in the EEG were classified according with the age in the following manner: 42.25 per cent in the first group, 33.75 per cent in the second group, and 16 per cent in the third group. 
5. These patients were distributed according to age of the first seizure: 30.20 per cent in the group from zero to 10 years, 41.50 per cent in the group from 11 to 20 years, 31.9 in the group from 21 to 35 years, and 8.5 per cent in the group with more than 36 years.

6. The patients with non epileptic changes in the EEG could be classified: 17.94 per cent in the first group, 29.5 per cent in the second and 51.7 per cent in the third.

7. These patients were classified according to the first seizure in: 15.9 per cent in the group from zero to 10 years of age, 27.5 per cent in the group from 11 to 20 years of age, 39 per cent in the group from 21 to 35 years of age and 59.7 per cent in the group with more than 36 years of age.

8. The EEG changes and the topography of these changes, when they were present, were coincident with the clinical syndrome in high percentage.

9. Bravais-Jacksonian seizures had negative EEG in high percentage of cases (36 in 91), in discordance with the other types of focal seizures. Temporal seizures were negative in 10 among 132 cases, and the other motor seizures with lateralization were negative in 18 among 125 cases.

10. GM seizures were the type with higher incidence of negative EEG (236 among 572 cases). PM seizures indicated the diagnosis of endogenous lesion ( 43 in 4.4 cases).

11. Temporal seizures presented lesional type of changes in the EEG in high percentage of cases (118 among 132 cases). These lesional type of changes were of focal type in temporal lobes in 86 among 118 cases.

12. The other types of motor seizures with lateralization showed high incidence of lesional changes in the EEG (88 among 127 cases).

13. The incidence of epilepsy among relatives was higher in the group of endogenous epilepsy (90 in 219 cases).

14. The neuro-psychiatric signs of cerebral organic diseases were coincident with lesional type of changes in the EEG in high incidence (103 among 130 patients).

15. The barbituric sleep was used when the routine EEG or the EEG with hyperventilation did not show evident changes. In this way it was possible to bring out positive findings in 65 among 202 cases. The barbituric sleep was helpful in 65 cases of temporal seizures and negative in 9.

16. The metrazol was used in 65 patients, with the same criteria. It was helpful in 46. 
17. The majority of the 307 patients with EEG showing no epileptic patterns was older than 35 years. This probably indicates the existence of other co-factors (as chronic intoxications, anoxia due to cerebral arteriosclerosis, hypoglycemia, etc.), when cerebral lesions are not present, in the genesis of paroxistic disturbances.

\section{BIBLIOGRAFIA}

1. Pupo, P. P. - O conceito da Escola Neurológica de Montreal sôbre a epilepsia. Arq. Neuro-Psiquiat., 8:257-270 (setembro) 1950. 2. Longo, P. W.; Pupo, P. P.; Pazzanese, O. - Diagnóstico e orientação terapêutica na epilepsia. Rev. Paulista de Med., 39:417-439 (novembro) 1951. 3. Pupo, P. P.; Pazzanese, O.; Sampaio, B. A. - Convulsões na infância. Considerações a propósito de 231 pacientes com diagnóstico clínico e eletrencefalográfico. Ped. Prát. (São Paulo), 23: 231-255 (novembro-dezembro) 1952. 4. Kershman, J.; Vásque\%, J.; Goldstein, S. - The incidence of focal and nonfocal abnormaiities in clinical epilepsy. EEG.

a. Clin. Neurophysiol., 3:15-24, 1951. 